\title{
Comparison of Constrained Parameterisation Strategies for Aerodynamic Optimisation of Morphing Leading Edge Airfoil
}

\author{
Andrea Magrini ${ }^{1,2, *,+}$ (D), Ernesto Benini ${ }^{1,2}{ }^{(0)}$, Rita Ponza ${ }^{2}$, Chen Wang ${ }^{3}$ (D), \\ Hamed Haddad Khodaparast ${ }^{3}$ D , Michael I. Friswell ${ }^{3}$ (D), Volker Landersheim ${ }^{4}$, \\ Dominik Laveuve ${ }^{4}$ and Conchin Contell Asins ${ }^{4}$ \\ 1 Department of Industrial Engineering, University of Padova, 35131 Padova, Italy; ernesto.benini@unipd.it \\ 2 HIT09 s.r.l., 35137 Padova, Italy; r.ponza@hit09.com \\ 3 College of Engineering, Swansea University, Swansea SA1 8EN, UK; chen.wang@swansea.ac.uk (C.W.); \\ h.haddadkhodaparast@swansea.ac.uk (H.H.K.); m.i.friswell@swansea.ac.uk (M.I.F.) \\ 4 Fraunhofer Institute for Structural Durability and System Reliability LBF, 64289 Darmstadt, Germany; \\ volker.landersheim@lbf.fraunhofer.de (V.L.); dominik.laveuve@lbf.fraunhofer.de (D.L.); \\ conchin.contell.asins@lbf.fraunhofer.de (C.C.A.) \\ * Correspondence: a.magrini@hit09.com \\ † Current address: Piazzetta Bettiol 15, 35137 Padova, Italy.
}

Received: 31 January 2019; Accepted: 28 February 2019; Published: 6 March 2019

\begin{abstract}
In the context of ambitious targets for reducing environmental impact in the aviation sector, dictated by international institutions, morphing aircraft are expected to have potential for achieving the required efficiency increases. However, there are still open issues related to the design and implementation of deformable structures. In this paper, we compare three constrained parameterisation strategies for the aerodynamic design of a morphing leading edge, representing a potential substitute for traditional high-lift systems. In order to facilitate the structural design and promote the feasibility of solutions, we solve a multi-objective optimisation problem, including constraints on axial and bending strain introduced by morphing. A parameterisation method, inherently producing constant arc length curves, is employed in three variants, representing different morphing strategies which provide an increasing level of deformability, by allowing the lower edge of the flexible skin to slide and the gap formed with the fixed spar to be closed by a hatch. The results for the optimisation of a baseline airfoil show that the geometric constraints are effectively handled in the optimisation and the solutions are smooth, with a continuous variation along the Pareto frontier. The larger shape modification allowed by more flexible parameterisation variants enables an increase of the maximum lift coefficient up to $8.35 \%$, and efficiency at $70 \%$ of stall incidence up to $4.26 \%$.
\end{abstract}

Keywords: morphing leading edge; constrained parameterisation; aerodynamic optimisation

\section{Introduction}

In the frame of increasingly stringent regulations governing the air transport sector [1], driven by environmental and economic concerns, continuing efforts are being made to reduce air traffic fuel consumption, pollutants, and noise emissions. To tackle the impact of the ever-growing volume of air traffic, with the number of transported passengers expected to increase by a factor of 7 by 2050 [2], international institutions set ambitious targets. This encourages aircraft manufacturers to develop innovative solutions for future vehicles, looking at novel configurations and technologies. Among these, deformable or morphing structures which are able to match the vehicle's state to the environment, in order to increase its performance, have been studied over the last twenty years. 
Now, morphing is recognised as a technology with the potential of an effective improvement of flight efficiency by continuous adaptation of wing shape to external conditions. It can involve a change of the wing section, wing plane, or an out-of-plane deformation [3], but a general classification has still to be agreed upon [4].

Among the available strategies, a compliant variation of leading or trailing edge represents the natural morphing counterpart to the traditional high-lift devices that are normally used at take-off and landing. Although effective in increasing lift in these flight phases, discrete devices have been recognized as one of the main sources of airframe noise [5]. Moreover, they enhance turbulence and promote boundary layer transition, even in the retracted position [6], penalising the efficiency at cruise and preventing laminarization. Conversely, a gapless device like a morphing leading edge, despite not providing the same lift increase of a slotted slat [7], does not affect cruise performance and possibly enables optimal operation at different flight conditions [8].

The morphing leading edge concept has received a fair amount of attention from the scientific community in the past few years, and has been investigated in several European projects like SADE [9,10], SARISTU [11], NOVEMOR [12], LeaTop [13], and the Green Regional Aircraft platform of Clean Sky [14], where structural optimisation, material selection, actuation mechanism design, and integration aspects were assessed. In fact, despite the fact that the main reason to employ this technology is the potential aerodynamic benefit, structural issues represent the main challenge for its adoption. In order to identify a set of shapes that can be actually reproduced by a structural morphing and fully exploit its potential, a multi-disciplinary approach should be followed, where the system is considered as a whole and in early stage of the design [15].

In practice, an aerodynamic optimisation is frequently used to produce a target shape, upon which the structure is subsequently optimised. However, a crude optimisation can pose a serious feasibility issue, if the reference geometry is extremely difficult to be matched. For this reason, the structural requirements need to be somehow considered in the aerodynamic optimisation; for example, by using a parameterisation that replicates the actual airfoil deformability $[6,16]$ or limiting the length and curvature variation [17] of candidate solutions. A more integrated approach is reported in [18], where concurrent aero-structural optimisation was performed in a multi-disciplinary framework. More recently, [19] employed a multi-disciplinary optimisation (MDO) for a morphing trailing edge of a regional aircraft, where constraints included size parameters and maneuver stress. A static aeroelastic coupling is described in [20], for the Fish Bone Active camber morphing of Swansea University.

For a more standard droop-nose leading edge, where the deformable skin extends up to some fraction of the chord, typically from $10 \%$ to $20 \%$, and the mechanism is expected to provide sufficient stiffness when locked, a full aeroelastic optimisation might not be necessary and proper introduction of structural limits in the aerodynamic analysis is expected to produce a target shape that can be reasonably obtained by optimisation of skin stiffness, actuation forces, and load application points. Aerodynamic loads are included in the structural design and a static aeroelastic analysis can, then, be used as an additional post-check verification.

The scope of this paper is to investigate suitable methods for the aerodynamic design of a morphing leading edge, by employing a constrained shape parameterisation for the aerodynamic optimisation, including structural constraints to limit the stress induced by the deformation. The study reported is part of the funded CleanSky2 OPTIMOrph project, addressing methods for optimisation of morphing structures, including specific mechanical boundary conditions. Despite that a full multi-disciplinary approach can account for all the aspects of actual technology implementation, effectively quantifying the impact of active compliant structures at different flight phases, especially for novel configurations or full span morphing, this paper is focused on the conceptual level evaluation of a morphing leading edge, employed as a substitute for traditional high-lift devices, and is aimed at identifying a procedural approach for its design.

Here, we present variants of a parameterisation strategy that tries to limit the structural stress introduced by morphing by employing a constant arc length (CAL) shape generation and including 
a local curvature variation constraint in the optimisation. We seek to use a technique that is able to generate individuals automatically, respecting the length constraints and also replicating the actual morphing path. Introduction of such limitations in the set of candidate solutions has the aim of facilitating the successive structural design, providing a more reliable quantification of the effective capability of replacing traditional slats by means of a compliant structure. In fact, despite the potential benefits of a morphing leading edge described above, the extent of the shape change cannot guarantee the same level of lift increase, compared to standard devices. By analysing three variants within the same parameterisation framework, we show that it is possible to enhance the airfoil deformability while fulfilling geometric constraints, thus allowing for a larger gain in the aerodynamic optimisation.

The rest of the paper presents the optimisation problem, the methods for objectives evaluation, and the parameterisation technique employed; reporting results for two cases and comparing them, in light of the three variants adopted.

\section{Optimisation Framework}

In order to check different formulations for the aerodynamic design of the morphing leading edge, we set up an optimisation framework to be applied to a starting geometry to find optimal morphed solutions. The undeformed reference shape was chosen to be a NACA 65(3)-218 airfoil, shown in black in Figure 1, whose experimental characteristics are known [21]. The rest of the section illustrates the various components of the optimisation loop, starting from the problem formulation.

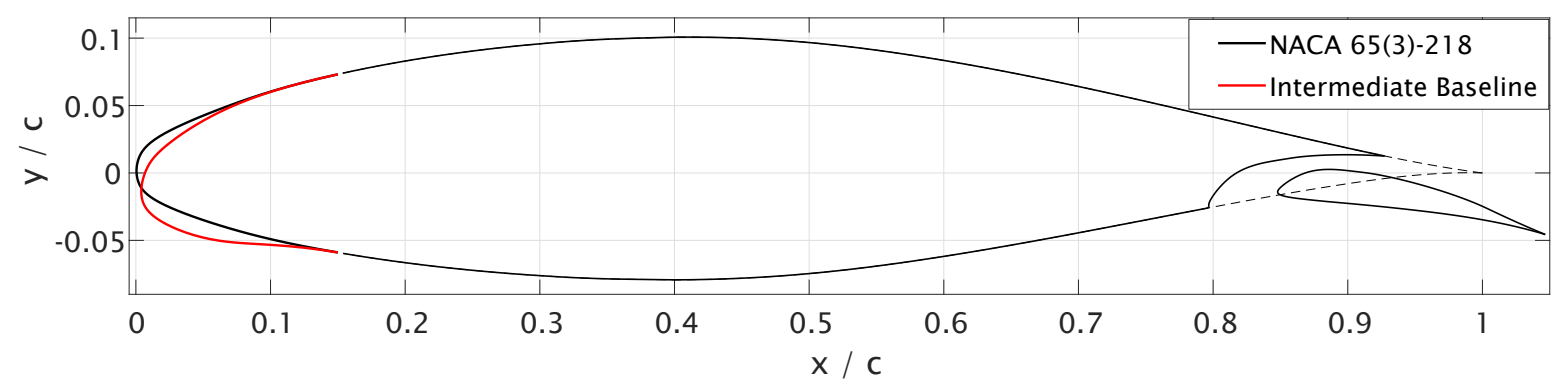

Figure 1. Reference shapes considered in the optimisation. Morphing region extends up to $0.15 x / c$. In black is a NACA 65(3)-218 airfoil, used as baseline in a first optimisation. In red is an intermediate baseline, used as a better starting point in the following optimisations (see Section 3.1). The original trailing edge of the NACA profile is drawn with a dashed line, the slotted flap with a solid line.

\subsection{Problem Formulation and Objectives Evaluation}

Typical objectives of optimisation for morphing include maximisation of lift to drag ratio at constant $C_{l}[16,20]$, or with $C_{l}$ as a constraint [22], and also $C_{d}$ minimisation [23-25]. As the purpose of the study was to investigate the high-lift performance of a compliant droop-nose, we defined a multi-point and multi-objective optimisation seeking to maximise the performance at take-off and landing, in terms of maximum lift coefficient and aerodynamic efficiency:

$$
\text { minimise }\left\{\begin{array}{l}
-C_{l, \max } \\
-L / D @ 0.7 C_{l, \text { max }},
\end{array}\right.
$$

with Mach number 0.15 and Reynolds number $9 \times 10^{6}$. Aerodynamic and geometric constraints were added to the problem and treated with the penalty function approach (subscript 0 refers to a reference condition):

$$
\text { minimise }\left\{\begin{array}{l}
C_{l, \max }>=C_{l, \max 0} \\
\left|L-L_{0}\right| / L_{0}<1 \times 10^{-6} \\
\max \left|\kappa-\kappa_{0}\right|<20(1 / m) .
\end{array}\right.
$$


The first ensures that only individuals with a lift coefficient higher than the baseline one are selected, the others are geometric constraints that restrict the axial and bending deformation introduced by the morphing and facilitate the structural design. A similar approach is described in $[17,26]$, where curve elongation was avoided and maximum local curvature variation, related to bending deformation, was limited. The threshold value of $20(1 / \mathrm{m})$ was derived from analysis of allowable curvature versus bending stress for a Fiber-reinforced Plastic (FRP) structure, optimized for bending in morphing applications.

For the evaluation of aerodynamic performance, we used a validated Computational Fluid Dynamics (CFD) model employing commercial software: Pointwise for mesh generation and the finite volume solver ANSYS Fluent. The validation activity involved the choice of turbulence model and an assessment of sensitivity to mesh topology and size. Turbulence model was first selected by comparing the numerical results of lift and drag polars with experimental data for NACA 65(3)-218. Figure 2a shows the $C_{l}(\alpha)$ curve obtained experimentally and numerically, with four CFD turbulence models, Spalart-Allmaras (SA) [27], $\kappa-\omega$ SST [28], $\kappa-\omega$ SST $\gamma$-Transition [29], and $\gamma-\operatorname{Re}_{\theta}$ [30], and a well-known panel-based code, XFOIL [31]. All the CFD models gave similar results up to $\alpha=10^{\circ}$, but $\kappa-\omega$ SST provided a better estimation of curve trend at higher incidence. Instead, XFOIL was unable to capture the stall, due to the limitations inherent to its approach. In terms of drag coefficient, Figure $2 \mathrm{~b}$ shows that the fully-turbulent models $\kappa-\omega$ SST and SA had the same prediction at low $\alpha$, whereas transitional CFD models offered an advantage at moderate incidence and outside the laminar bucket. In that region, only XFOIL correctly reproduced the experimental curve. Given the interest to study the high-incidence behaviour of the morphing leading edge, $\kappa-\omega$ SST was selected, due to its capacity to better represent the reference results in that condition.

(a)

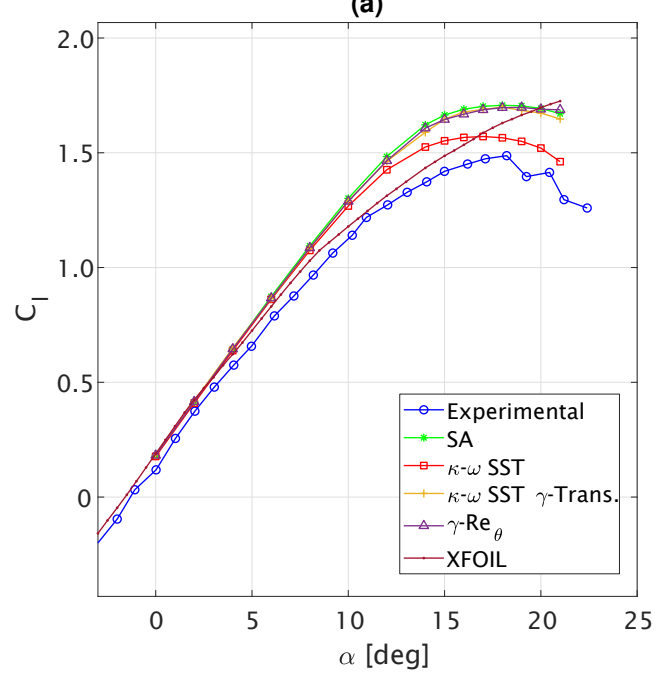

(b)

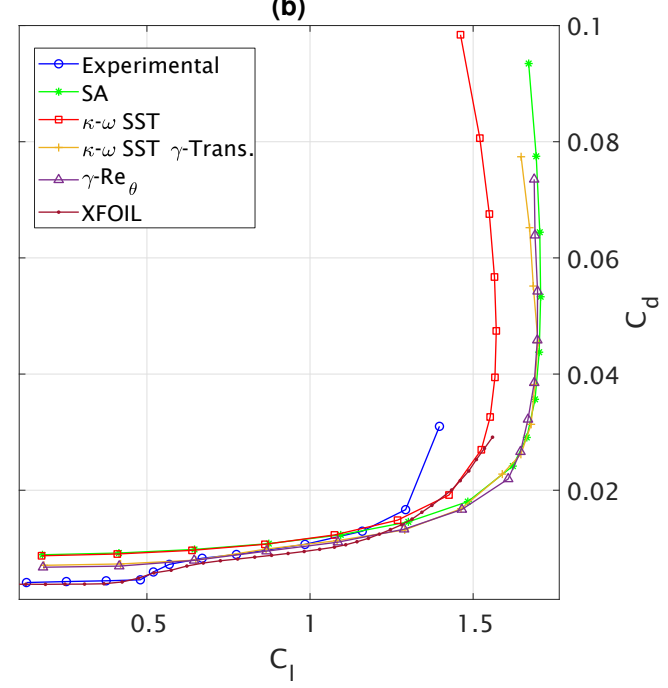

Figure 2. Comparison of lift (a) and drag (b) polars with different turbulence models.

In the second phase of the validation, the grid dependency was more deeply studied by assessing the influence of topology (O-grid versus $\mathrm{C}$-grid), far-field radius, and number of points per airfoil side. The O-grid topology was chosen over a C-grid for the higher flexibility in meshing general shapes in a fully automatic way, a very small difference between them in predicted values and a lower number of cells required. In general, it was difficult to obtain a complete correspondence with experimental data, regardless of the mesh employed. A parametric study of farfield radius of grids with different number of cells (from 55k to 200k) revealed that the error in maximum lift coefficient was less sensitive to cell number for wider domains and could be reduced by decreasing domain size. In Figure 3a, the best result for the relative percentage error on $C_{l, \max }$ at different farfield radii is reported. The final selected model was the one giving the lowest error on stall $C_{l}$, equal to $2.75 \%$, with a farfield radius of 25 chord length, 240 points per airfoil side, and a total of 100k cells. Like all other cases examined, 
the first wall spacing was chosen to obtain $y^{+} \approx 1.0$. Hyperbolic extrusion was used to provide wall orthogonality over the whole airfoil, with an expansion ratio of 1.06. The maximum aspect ratio was 2500 , in the boundary layer region. The minimum included angle was above $88.5^{\circ}$ for $98.5 \%$ of cells, with a minimum value of $30^{\circ}$ near the trailing edge. The validated lift and drag polars are depicted in Figure $3 b$.
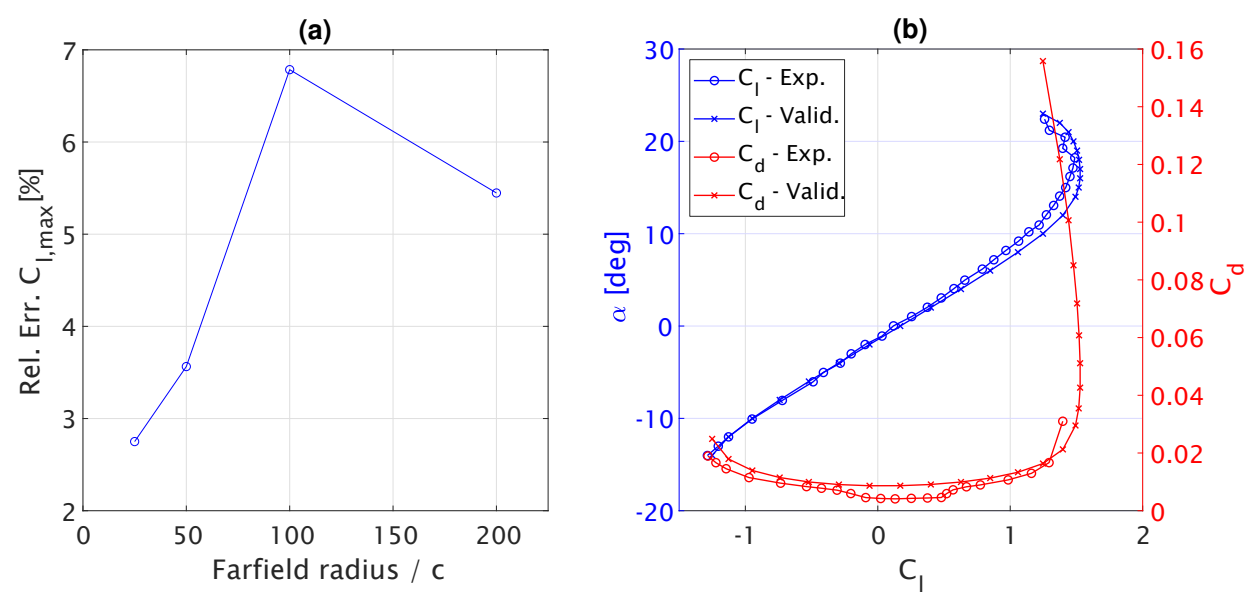

Figure 3. Best obtained relative percentage error in maximum lift coefficient for different farfield radii (a). Validated lift and drag polars (b).

Computation of objectives requires the identification of stall condition, hereafter denominated as design point DP1, and incidence where $C_{l}$ is $70 \%$ of the maximum value, indicated as DP2. A conditional grid search with $1^{\circ}$ resolution around baseline stall incidence was used to find DP1, while at DP2 the Regula Falsi algorithm was chosen and convergence to the right incidence within a $1 \%$ tolerance on $C_{l}$ was achieved in three iterations at most, thanks to a judicious choice of starting point. This led to an average total number of CFD calls per individual evaluation of 4.63 in the first optimisation.

The optimisation method used was GeDEA-II [32], an in-house multi-objective genetic algorithm implementing modified genetic operators which inherently drive the search towards feasible optima and includes genetic diversity as a real objective. To this purpose, GeDEA-II proved unique in its capabilities for handling multiple constraints and numerical tests conducted on highly multimodal functions with multiple constraints revealed algorithm superiority, with respect to other evolutionary strategies [33].

\subsection{Parameterisation Strategy}

The final component of the optimisation loop is the parameterisation technique. It is known that it has a large impact on the obtained solution and it should be flexible enough to cover the whole design space, produce smooth shapes, allow a local control of geometrical parameters, and provide a small set of coefficients with, possibly, a direct physical meaning [34]. Various techniques are reported in the literature for morphing problems, including polynomials [35], Radial Basis Function (RBF) [16], Class/Shape Transformation (CST) [17,36], and Free Form Deformation (FFD) [19].

In our previous work, we also used the CST technique [37] where a novel strategy allowed generation of curves attaining a constant arc length to reduce the induced axial strain, applying both multi-objective and multi-point optimisation to different problems, using a genetic algorithm and a hybrid optimisation strategy with metamodel assistance [38]. Despite the good results obtained, we aim to overcome some limitations of the previous approach that made it less suitable for high-lift optimisation. To this end, we redefined the parameterisation method relying on cubic B-Splines. Contrary to CST, where the whole airfoil is described by a polynomial function representing a generalisation of Bézier curves, and least-square minimisation is employed to confine the morphing 
to the front part of the airfoil, B-Splines enable a more intuitive local deformation through control point movement. The CAL attainment procedure followed the work of Bettadapura [39], and was originally intended for Bézier curves. However, due to their similar definition, it was applied first to CST, as fully described in [38], and it could be reused also for B-Splines. Thanks to this algorithm, the CAL requirement is inherently satisfied, whenever it is possible, every time a new individual is generated by the optimiser.

The baseline length was chosen to be the arc length of the reference shape within the first $15 \%$ of the chord, corresponding to the morphing region; the remainder of the wing being fixed. The CAL attainment requires a further degree of freedom to be invoked to recover the unperturbed length, once a modification of the curve is introduced. It is based on an iterative procedure where the non-linear relationship between the global arc length and the displacement of a specified control point, representing a dependent variable, is repeatedly locally approximated. The advantage of B-Spline parameterisation over CST for our problem is that there is a better local control of the curve, only the deformable part is represented, the chord can be changed without any problem, and the movement of a control point can produce only a localised variation. These aspects are particularly useful since they allow a certain flexibility in the definition of the control points (CP) and, in particular, in the choice of which of them have to be moved according to the CAL procedure.

The first formulation tested, named variant $A$, used ten control points to fully represent the deformable skin with a cubic B-Spline, whose edges lay in correspondence of a normalized abscissa $x / c=0.15$. $C(1)$, and continuity was ensured by fixing a pair of consecutive points on each edge. Nine design variables (DV) were used to specify the $x$ - and $y$-displacements of moving CPs, whose bounding box is shown in Figure 4a. The ordinate of point, indicated as P8, instead, was a dependent variable found through the CAL procedure. Its positioning on the pressure side alleviates potential undesirable effects, due to its displacement being not directly settable. In fact, for some excessive deformation, the unperturbed length cannot be recovered any more and warped individuals could be generated. An active bound on P8 displacement guarantees that, even in that condition the resulting shape is smooth enough to be simulated by CFD, its fitness will be penalised as described above.

The second formulation, named variant $\mathrm{B}$, aimed at providing more deformability by alleviating the $C(1)$ constraint on the lower edge of the curve. The B-Spline now has nine CPs, and ends at point P9 in Figure 4b, which is moved horizontally according to the CAL procedure. In this configuration, the lower edge can slide to provide extra flexibility (e.g., to increase chord length), while fulfilling the geometric constraint. The gap created with the fixed part of the airfoil is closed by a straight hatch connecting the two edges. Eleven DVs fully describe each morphed geometry.

In the third variant $C$, similarly to the previous one, point P9 in Figure $4 \mathrm{c}$ can slide along a specified direction, $\theta$, measured counter-clockwise from the positive $x$-axis. The direction $\theta$ is a twelfth DV and was introduced since, due to the straight hatch, two corners appears at its edges: By changing the displacement direction of $\mathrm{P9}$, it is possible to achieve higher smoothness and possibly larger deformability. The extent of the displacement was again found with the CAL procedure. 
(a)

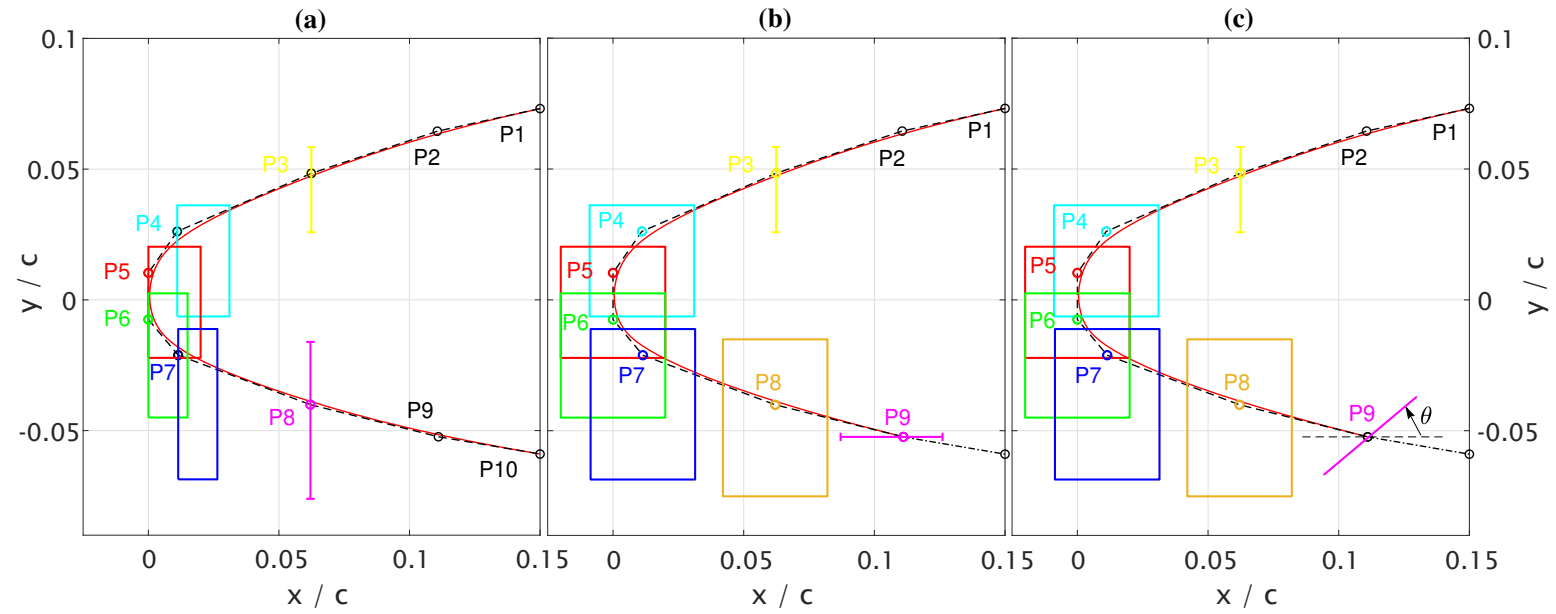

Figure 4. Parameterisation variants and bounding box of control points. Control points with a black labels are fixed, moving control points and their corresponding bounding box are plotted in the same colour. (a) Variant A: Spline edges are fixed and P8 is moved with the Constant Arc Length (CAL) procedure. (b) Variant B: Lower edge P9 is moved horizontally with the CAL procedure, and the resulting gap is closed with a straight hatch. (c) Variant C: The lower edge P9 slides along an optimiser-controlled direction $\theta$, and resulting gap is closed with a straight hatch.

\section{Results and Discussion}

The optimisation framework was applied to two main cases, using the three variants described above. In the first case, the fixed region corresponded to the NACA 65(3)-218 airfoil with a clean trailing edge, shown as dashed in Figure 1. The objective of maximisation of lift coefficient, required to bring the airfoil to stall, happened at $17^{\circ}$ for NACA profile. That high incidence condition appeared demanding for the optimiser, which was expected to exploit the whole bending margin in order to reduce the angle between the chord tangent at nose and the flow direction. In this way, the constraint handling effectiveness can be tested and also related to the actual achievable lift coefficient.

In a second case, the same problem was applied with a slotted flap in the trailing edge, as shown in Figure 1. This condition was more realistic, and by reducing the stall angle of attack could enable the morphed leading edge to better adapt to flow direction, given the deformability constraint.

In the following, the results obtained for the two cases are reported and discussed, comparing the parameterisation variants adopted.

\subsection{Optimisation without Flap}

The optimisation framework was first applied to the clean NACA 65(3)-218, as reference airfoil, using the parameterisation Variant A and finding a set of Pareto optimal solutions. As the potential of morphing is to allow the adaption of the nose shape to different flight conditions, we defined a new baseline having a shape in an intermediate position between the undeformed initial geometry, which was found to be near optimum for minimum drag with target $C_{l}$ at cruise condition, and the best compromise Pareto airfoil. This new baseline airfoil, hereafter denoted as intermediate and depicted in red in Figure 1, belonged to a partial converged front and was then reused as reference shape in a second run, where the population size was 30 and total number of generations was 50 . Being partially bent, it represented a better starting point which provided further deformability. The optimisation was also extended to Variants B and C.

The normalized Pareto frontiers obtained are shown in Figure 5, from which a further objective reduction, when enabling additional deformation paths, is evident. Table 1 summarizes the change in aerodynamic coefficients for airfoils at the edges of the curves, reporting relative percentage variations, with respect to the intermediate baseline. The extra deformability provided by Variants B and $C$ led to higher maximum lift coefficient in all the cases considered. Reaching a greater $C_{l, m a x}$ also increases 
$C_{l}$ at DP2, which is the main reason for obtaining a better $L / D$ ratio, since a slight drag growth was compensated by a larger lift. It can also be noticed that, apart from Variant $C$, efficiency improved at both design points for the best $L / D$ airfoils. For the opposite case of Figure 6a, the use of a moving lower edge allowed for more than double the stall $C_{l}$ relative gain at DP1, from $1.82 \%$ for Variant $A$ to $4.07 \%$ for Variant C. Compared to the clean NACA geometry, the variation in $C_{l, \max }$ was up to $7.25 \%$ for Variant A and $9.61 \%$ for Variant C.

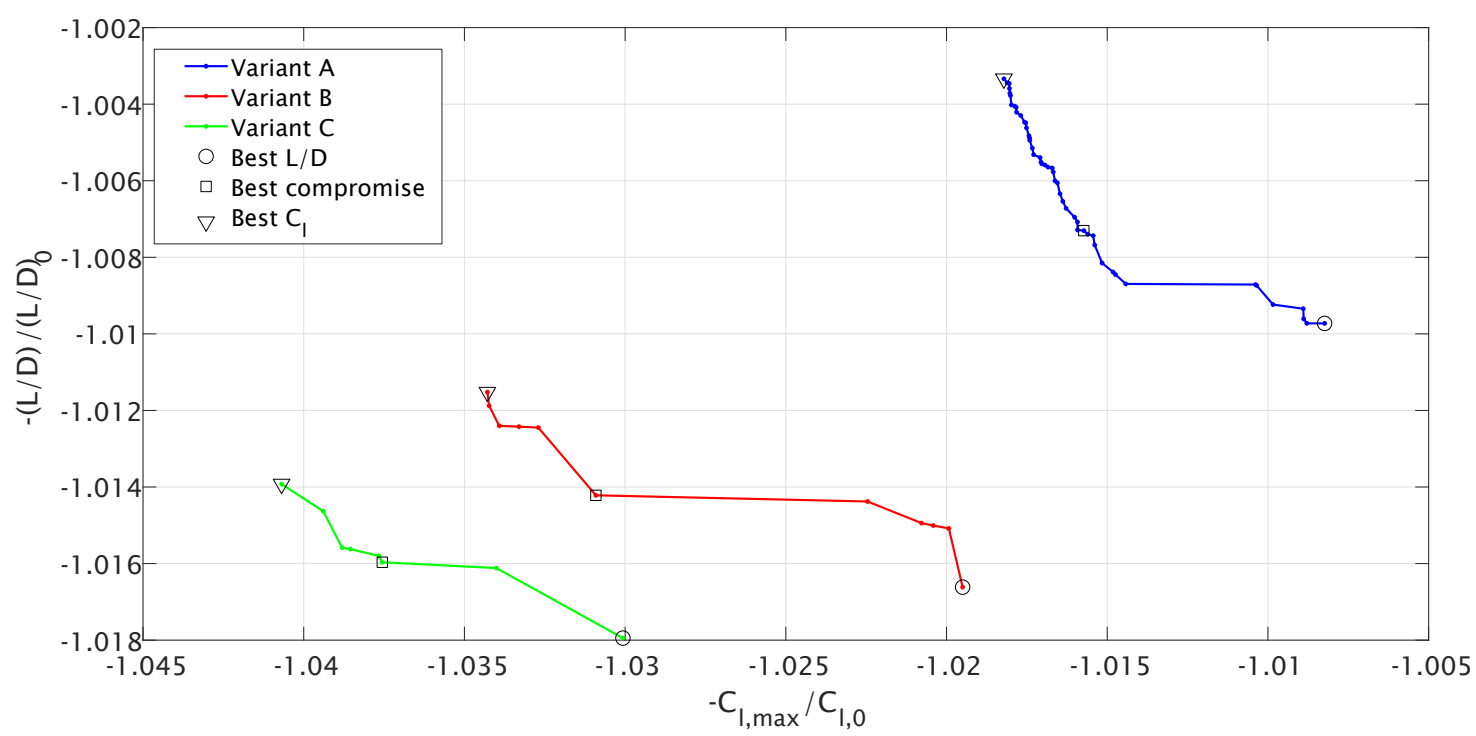

Figure 5. Pareto frontiers obtained for case without flap, using three parameterisation variants. Objectives are normalized with respect to intermediate baseline. Selected points on Pareto corresponding to best improvement of $C_{l, \max }, L / D$, and best compromise are also marked.

Table 1. Comparison of aerodynamic performance of optimised individuals with three parameterisation variants. $C_{d}=$ drag coefficient, $C_{l}=$ lift coefficient, $L / D=$ lift to drag ratio. Between parenthesis are the percentage relative variations, with respect to the intermediate baseline.

\begin{tabular}{ccccc}
\hline & DP1 & & DP2 \\
\hline Airfoil & $C_{\boldsymbol{d}}$ (rel. var. \%) & $\boldsymbol{C}_{\boldsymbol{l}}$ (rel. var. \%) & $\boldsymbol{\alpha}$ & $\boldsymbol{L} / \boldsymbol{D}$ (rel. var. \%) \\
\hline NACA 65(3)-218 & $0.04766(-2.22)$ & $1.571(-5.07)$ & 17.00 & $80.42(-2.37)$ \\
Interm. Baseline & 0.04874 & 1.655 & 18.00 & 82.37 \\
Best L/D-var. A & $0.04722(-3.11)$ & $1.669(+0.82)$ & 18.00 & $83.17(+0.97)$ \\
Best L/D-var. B & $0.04663(-4.33)$ & $1.687(+1.95)$ & 18.00 & $83.74(+1.66)$ \\
Best L/D-var. C & $0.05400(+10.80)$ & $1.705(+3.00)$ & 19.00 & $83.85(+1.79)$ \\
Best Cl一var. A & $0.05390(+10.58)$ & $1.685(+1.82)$ & 19.00 & $82.65(+0.33)$ \\
Best Cl-var. B & $0.05280(+8.33)$ & $1.712(+3.43)$ & 19.00 & $83.32(+1.15)$ \\
Best Cl-var. C & $0.05252(+7.75)$ & $1.722(+4.07)$ & 19.00 & $83.52(+1.39)$ \\
\hline
\end{tabular}

Selected airfoils on three Pareto fronts obtained, corresponding to best $L / D$, best compromise, and best $C_{l}$ cases of Figure 5, are depicted in Figure 6. By analysing the results for the first variant, a smooth and continuous variation can be observed for airfoil shapes, with rightmost Pareto individuals (Figure 6c) featuring a smaller nose radius and a slight pressure side concavity. Proceeding towards higher maximum lift coefficients, the nose becomes rounder and the downward bending larger. A similar trend can be observed for Pareto airfoils obtained with Variant B, shown in red in Figure 6, and also for Variant $C$, shown in green; even though, in the latter case, with a lower variability and a thicker nose. 
(a)

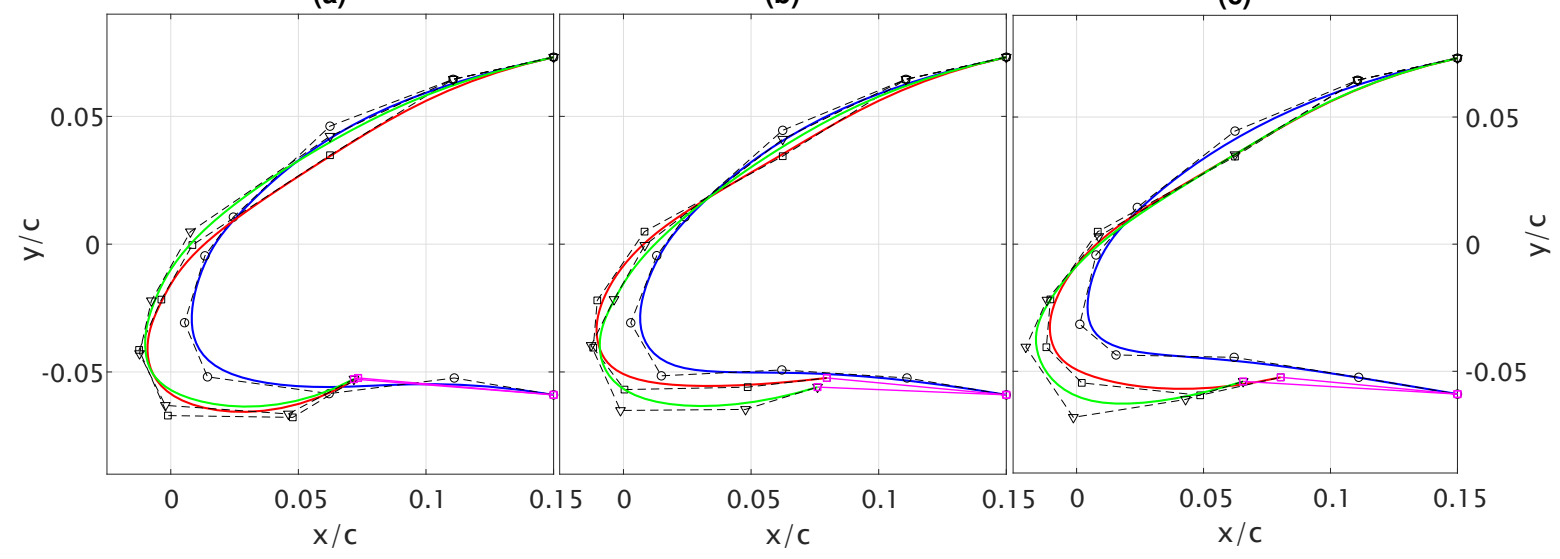

Figure 6. Pareto airfoils for three parameterisation variants, corresponding to marked points on Figure 5. Control polygon represented with a black dashed line, straight hatch with a solid magenta line. Blue: Variant A; red: Variant B; green: Variant C. (a) Best $L / D$. (b) Best compromise. (c) Best $C_{l}$.

Distribution of pressure coefficients for best $C_{l}$ airfoils at DP1 is reported in Figure 7. The different chord makes the comparison of integral quantities less evident, but it can be observed that the major variation occured near the leading edge at upper side. The suction peak was progressively smoothed and redistributed from NACA airfoil to Variant $C$, with the formation of a region of lower pressure insisting on the morphing skin and gently degrading, up to mid chord. In Variants A and C, the kink following the suction peak was pronounced and followed by a region contributing to the total lift, thanks to an almost constant pressure. This is exacerbated in Variant B, having a straight upper side, where the curve was not monotonic and the initial pressure recovery was followed by a second flow acceleration generating a bubble, prior to the beginning of the fixed part of the airfoil. Further downstream, Variants $B$ and $C$ curves were very similar and slightly below the intermediate baseline from 0.4 to $0.62 x / c$, while Variant A had a lower $C_{p}$ compared to that baseline, up to the latter location. The corner at the pressure side, due to the hatch, seems to have a limited effect in the flow development in the model employed.

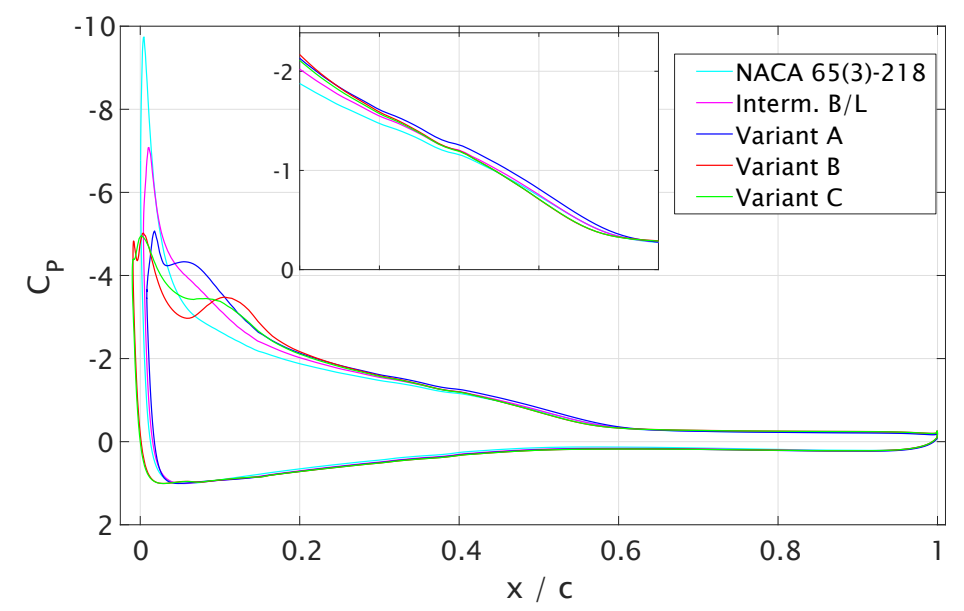

Figure 7. Pressure coefficient distribution at DP1 for baseline and best $C_{l}$ optimised airfoil, with three parameterisation variants.

The movement of point P9 led to an increase of the airfoil chord, conversely to the first variant where the chord decreased, resembling the deployment of a traditional slat. With regards to the difference between choosing a fixed or variable displacement direction for the point, a parametric study performed prior to the optimisation suggested that, in general, $\theta$ should be small. This was 
confirmed by the optimisation and the largest $\theta$ for Pareto individuals was $7.5^{\circ}$. We chose to keep only Variant $C$ for the next case, dealing with a trailing edge flap, due to the greater performance achievable.

\subsection{Optimisation with Flap}

The optimisation problem was also solved in the presence of a flap, to replicate a more realistic case, for the first and third strategies. The slotted flap, shown with a black solid line in Figure 1, was adapted to the NACA airfoil and had a fixed deflection angle of $15^{\circ}$, providing a stall $C_{l}$ of 2.25 for that airfoil. In this way, it was possible to keep the leading edge morphing effect predominant, and to make a fair comparison with the preceding result.

The initial population of the GA was partially filled with Pareto individuals from the previous run. Indeed, the airfoils at the edges of the frontier, shown in Figure 8, are very similar to those of Figure 6. The shape change across the front was, again, smooth, and Variants A and C produced resembling geometries, indicating that the lift increase mechanism is the same. This was confirmed by the pressure coefficient distribution at DP1, reported in Figure 9a, where curves show an attenuation of suction peak in morphed geometries, analogous to the previous case. Similarly, Mach number contours, illustrated in Figure 10 for the same geometries, show the spreading of the high-velocity area present in the clean baseline. The main variation occurs near the leading edge, but a slight thinning of the wake can be noticed in optimised airfoils.

(a)

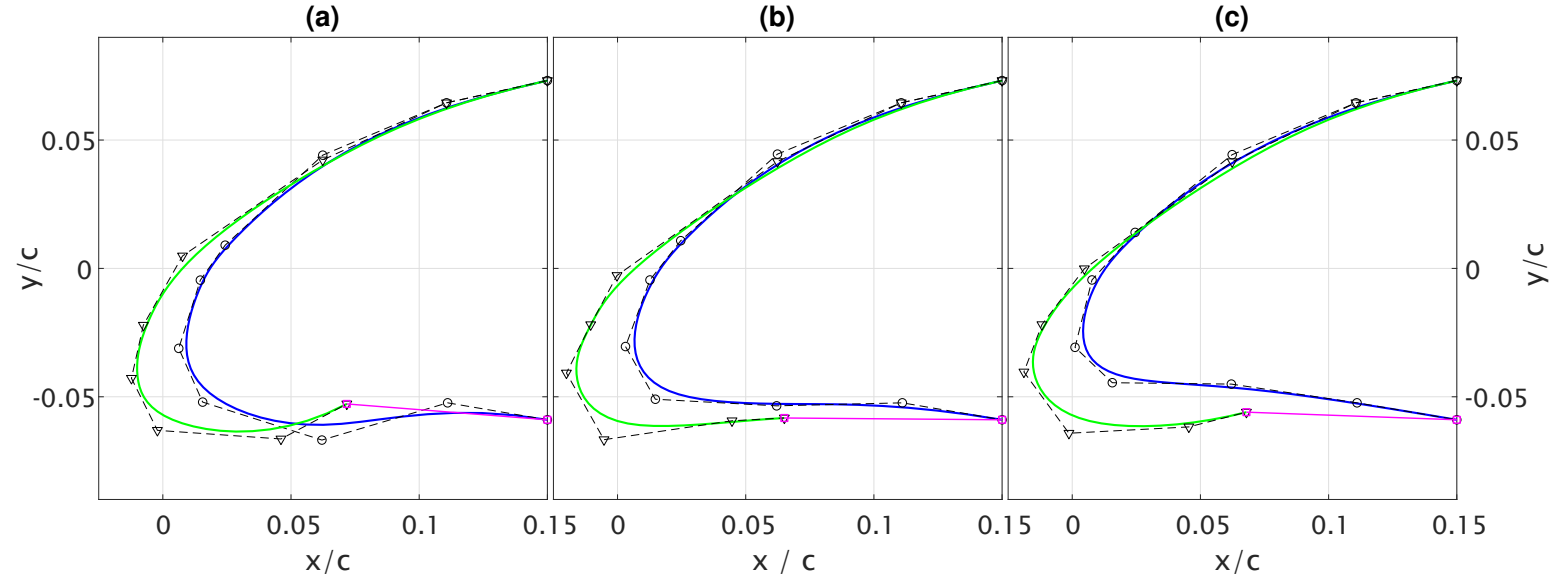

Figure 8. Pareto airfoils for three parameterisation variants. Control polygon represented with a black dashed line, straight hatch with a solid magenta line. Blue: Variant A; red: Variant B; green: Variant C.

(a) Best $L / D$. (b) Best compromise. (c) Best $C_{l}$.

Looking at the aerodynamic coefficients for selected Pareto individuals in Table 2, it can be observed that, at DP1, $C_{l}$ variation had an extent comparable to the no-flap case and the same holds for $L / D$ at DP2, for both variants. Differently, there was a substantial drag reduction at DP1, which was not achieved before, ameliorating the efficiency by $9.42 \%$ for Variant A and up to $13.72 \%$ for Variant C, at the left Pareto edge.

By inspecting how pressure and viscous forces contributed to the overall drag, the reason for this improvement was attributed mostly to pressure change at the leading edge. This is visible in Figure 9b, where pressure and viscous components at DP1 in different airfoil zones are plotted, for clean, intermediate baseline and the best $C_{l}$ optimised geometry. The major variation affecting the drag occurred for the pressure force in the airfoil, where its redistribution, caused by leading edge bending, produced a favourable effect, with a net reduction up to $73 \%$ for Variant $C$. The viscous part in the same zone, instead, progressively incremented, up to $24.5 \%$ for Variant $C$, compared to the NACA profile, while in the other zones it appeared similar for all the shapes. However, the viscous contribution to the overall drag at high incidence was small, as shown in the figure, amounting to $17 \%$ of $C_{d}$ on average, with higher values for optimised shapes. 
(a)

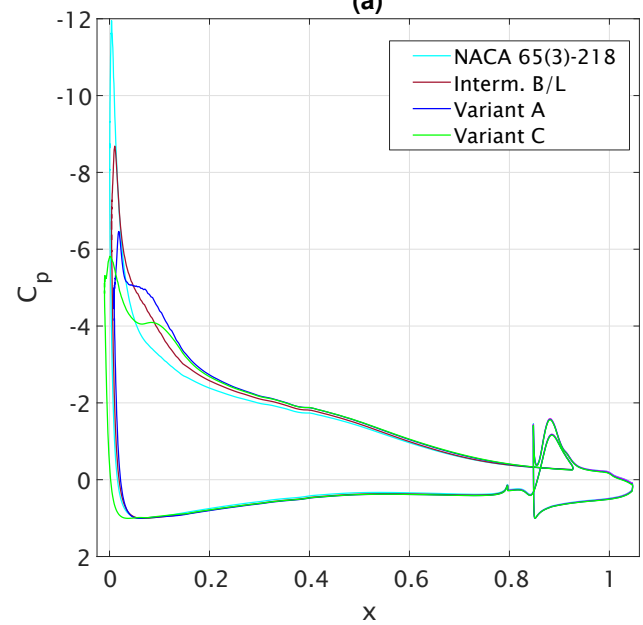

(b)

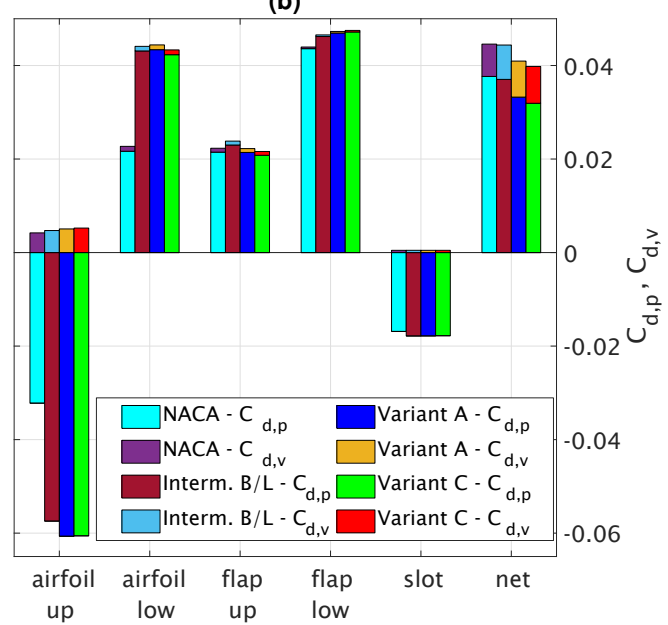

Figure 9. Pressure coefficient distribution at DP1 for baseline and optimised airfoil at left Pareto edge, with flap (a). Contribution of pressure and viscous drag at DP1 in different zones for the same airfoils (b).

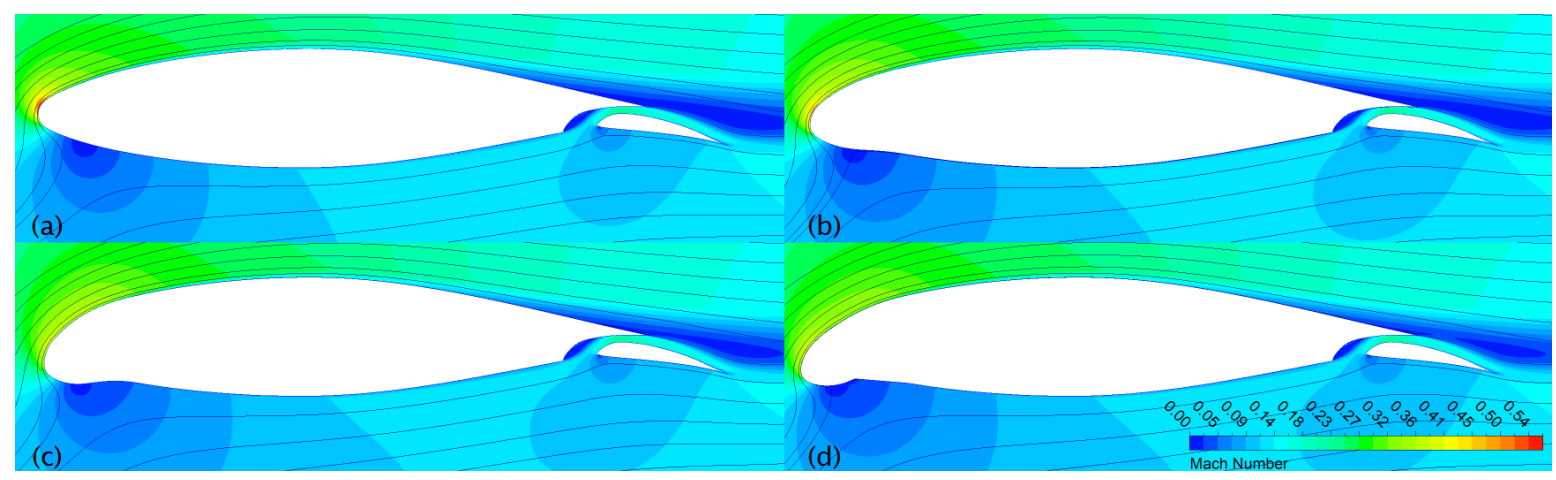

Figure 10. Mach number contour and flow path visualization at DP1. (a) NACA 65(3)-218. (b) Intermediate baseline. (c) Variant A-Best $C_{l}$. (d) Variant C-Best $C_{l}$.

The additional reduction of objective value, enabled by the looser formulation, was slightly lower than the previous result; in particular, for $C_{l, \max }$ of leftmost Pareto points, going from $2.26 \%$ to $1.74 \%$.

Table 2. Comparison of aerodynamic performances of optimised individuals with two parameterisation variants, in the presence of a trailing edge flap. Between parenthesis are the percentages of relative variations, with respect to the intermediate baseline.

\begin{tabular}{ccccc}
\hline \multicolumn{1}{c}{ DP1 } & & DP2 \\
\hline Airfoil & $C_{\boldsymbol{d}}$ (rel. var. \%) & $C_{l}$ (rel. var. \%) & $\alpha$ & $\boldsymbol{L} / \boldsymbol{D}$ (rel. var. \%) \\
\hline NACA 65(3)-218 & $0.04461(0.51)$ & $2.250(-4.64)$ & 14.00 & $82.30(-1.79)$ \\
Interm. Baseline & 0.04438 & 2.359 & 15.00 & 83.80 \\
Best L/D-var. A & $0.04253(-4.17)$ & $2.379(+0.85)$ & 15.00 & $84.40(+0.72)$ \\
Best L/D-var. C & $0.04047(-8.80)$ & $2.432(+3.07)$ & 15.00 & $85.23(+1.71)$ \\
Best Cl一var. A & $0.04092(-7.80)$ & $2.397(+1.62)$ & 15.00 & $84.04(+0.29)$ \\
Best Cl一var. C & $0.03978(-10.36)$ & $2.438(+3.36)$ & 15.00 & $84.99(+1.42)$ \\
\hline
\end{tabular}

In terms of displacement direction of P9, Figure 8 shows that it was, again, close to horizontal. Its distribution along the Pareto front had a similar trend for both optimisation cases, reaching a maximum of about $7.5^{\circ}$ near the centre, and rapidly decreasing while approaching the borders. The use of a variable direction could complicate the design of the actuation mechanism, especially if different target shapes are to be matched. However, a fixed moderate value of $\theta$ angle could well allow 
the reproduction of shapes in the middle of Pareto front, those that exhibit the best compromise in terms of objectives.

\title{
4. Conclusions
}

This paper presents a comparative study of parameterisation approaches for the aerodynamic optimisation of a morphing leading edge airfoil, providing an increasing level of deformability. Inclusion of structural limitations during the optimisation is mandatory to restrict the candidate solutions only to those that could be matched in reality by an actuation mechanism deforming the skin, especially when the boundary conditions require a large change in shape. The constraint handling strategy, based on a constant arc length parameterisation for curve generation and the use of an optimisation algorithm with inherent capabilities of driving the search towards feasible optima, appeared effective in producing shapes featuring a smooth variation along the Pareto frontier, which is advantageous, in light of the requirements of continuous adaptation to external conditions and full exploitation of morphing benefits.

The use of non-standard configurations with a movable lower edge of the flexible skin was also investigated, in order to provide extra deformability to the structure without violation of the constraints. Results of high-lift optimisation, also in presence of a trailing edge flap, showed that, in this case, it was possible to reach a larger increase in maximum lift coefficient and efficiency, both at stall and near peak point. Despite the advantages obtained, it is known that the performance gap with multi-element leading edge devices cannot be closed. Moreover, non-smooth connection in the lower side may hamper flow laminarisation, as in Kreuger flaps, partially limiting the benefits of a seamless skin, also, in terms of noise generation. In the fully turbulent flow model employed, it was not possible to analyse this aspect and the disturbance caused by pressure side corners appeared to be limited.

Finally, the presence of the hatch that must connect to the fixed part of the airfoil poses new challenges in the structural modelling, in terms of stiffness and actuation mechanism design. The additional system complexity should be compared against the achievable performance improvement, in order to establish the effective validity of the solution. The structural implications and possible implementation strategies will be evaluated in the more comprehensive frame of the OPTIMOrph project.

Author Contributions: Conceptualization, A.M., E.B., V.L., and M.I.F.; methodology, A.M., E.B., R.P., C.W., M.I.F., V.L., and D.L.; validation, formal analysis and investigation: A.M. and C.W.; supervision, H.H.K. and C.C.A.; writing—original draft preparation, A.M.; writing—review and editing, E.B., R.P., M.I.F., and V.L.; project administration, R.P., M.I.F., and V.L.

Funding: This research was part of the EU CleanSky2 OPTIMOrph project, funded under H2020-EU.3.4.5.4. GA no. 755068 .

Conflicts of Interest: The authors declare no conflict of interest.

\author{
Abbreviations \\ CAL Constant Arc Length \\ CFD Computational Fluid Dynamics \\ CP Control Point \\ DP Design Point \\ DV Design Variable \\ FRP Fiber-reinforced Plastic \\ MDO Multi-Disciplinary Optimisation \\ $\alpha \quad$ Angle of attack \\ c Airfoil chord \\ $C_{d} \quad$ Drag coefficient \\ $C_{l} \quad$ Lift coefficient
}

The following abbreviations are used in this manuscript: 
$C_{p} \quad$ Pressure coefficient

$\kappa \quad$ Curvature

$L \quad$ Arc length

$L / D \quad$ Lift to drag ratio

$\theta \quad$ Displacement direction

\section{References}

1. ACARE. Flightpath 2050. Europe's Vision for Aviation; Technical Report; European Commission, Directorate-General for Research and Innovation: Brussels, Belgium, 2011. [CrossRef]

2. IATA. IATA Vision 2050 Report; Technical report; International Air Transport Association: Montreal, QC, Canada, 2011.

3. Barbarino, S.; Bilgen, O.; Ajaj, R.M.; Friswell, M.I.; Inman, D.J. A Review of Morphing Aircraft. J. Intell. Mater. Syst. Struct. 2011, 22, 823-877. [CrossRef]

4. Li, D.; Zhao, S.; Ronch, A.D.; Xiang, J.; Drofelnik, J.; Li, Y.; Zhang, L.; Wu, Y.; Kintscher, M.; Monner, H.P.; et al. A review of modelling and analysis of morphing wings. Prog. Aerosp. Sci. 2018, 100, 46-62. [CrossRef]

5. Chow, L.; Mau, K.; Remy, H. Landing Gears and High Lift Devices Airframe Noise Research. In Proceedings of the 8th AIAA/CEAS Aeroacoustics Conference and Exhibit, Breckenridge, CO, USA, 17-19 June 2002. [CrossRef]

6. Kintscher, M.; Wiedemann, M.; Monner, H.P.; Heintze, O.; Kühn, T. Design of a smart leading edge device for low speed wind tunnel tests in the European project SADE. Int. J. Struct. Integr. 2011, 2, 383-405. [CrossRef]

7. Wild, J. Recent research topics in high-lift aerodynamics. CEAS Aeronaut. J. 2016, 7, 345-355. [CrossRef]

8. Ameduri, S. Chapter 16-Morphing of the Leading Edge. In Morphing Wing Technologies; Concilio, A., Dimino, I., Lecce, L., Pecora, R., Eds.; Butterworth-Heinemann: Oxford, UK, 2018; pp. 491-515. [CrossRef]

9. Kintscher, M.; Monner, P.; Kühn, T.; Wild, J.; Wiedemann, M. Low Speed Wind Tunnel Test of a Morphing Leading Edge. In Proceedings of the Italian Association of Aeronautics and Astronautics XXII Conference, Naples, Italy, 9-12 September 2013.

10. Monner, H.P. SADE Project Final Report; Technical Report; DLR: Köln, Germany, 2012.

11. Kirn, J.; Storm, S. Kinematic solution for a highly adaptive droop nose. In Proceedings of the ICAST 2014, Hague, The Netherlands, 6-8 October 2014.

12. De Gaspari, A. Design, Manufacturing and Wind Tunnel Validation of an Active Camber Morphing Wing Based on Compliant Structures. In Proceedings of the ICAST 2014, Hague, The Netherlands, 6-8 October 2014.

13. Sodja, J.; Martinez, M.; Simpson, J.; De Breuker, R. Experimental evaluation of the morphing leading edge concept. In Proceedings of the AIAA SciTech, 23nd AIAA/AHS Adaptive Structures Conference, Kissimmee, FL, USA, 5-9 January 2015.

14. Contell Asins, C.; Landersheim, V.; Schwarzhaupt, O. Development and Demonstration of a Multifunctional Morphing Leading Edge for a Regional A/C. In Proceedings of the Advanced Aircraft Efficiency in a Global Air Transport System AEGATS '18 Conference, Toulouse, France, 23-25 October 2018.

15. Ajaj, R.M.; Beaverstock, C.S.; Friswell, M.I. Morphing aircraft: The need for a new design philosophy. Aerosp. Sci. Technol. 2016, 49, 154-166. [CrossRef]

16. Fincham, J.; Friswell, M. Aerodynamic optimisation of a camber morphing aerofoil. Aerosp. Sci. Technol. 2015, 43, 245-255. [CrossRef]

17. De Gaspari, A.; Ricci, S. Knowledge-Based Shape Optimization of Morphing Wing for More Efficient Aircraft. Int. J. Aerosp. Eng. 2015, 2015, 325724. [CrossRef]

18. Molinari, G.; Quack, M.; Dmitriev, V.; Morari, M.; Jenny, P.; Ermanni, P. Aero-Structural Optimization of Morphing Airfoils for Adaptive Wings. J. Intell. Mater. Syst. Struct. 2011, 22, 1075-1089. [CrossRef]

19. Burdette, D.A.; Martins, J.R. Design of a transonic wing with an adaptive morphing trailing edge via aerostructural optimization. Aerosp. Sci. Technol. 2018, 81, 192-203. [CrossRef]

20. Woods, B.K.; Friswell, M.I. Multi-objective geometry optimization of the Fish Bone Active Camber morphing airfoil. J. Intell. Mater. Syst. Struct. 2016, 27, 808-819. [CrossRef]

21. Abbott, I.; Von Doenhoff, A. Theory of Wing Sections, Including a Summary of Airfoil Data; Dover Books on Aeronautical Engineering Series; Dover Publications: New York, NY, USA, 1959. 
22. Sun, R.; Chen, G.; Zhou, C.; Zhou, L.; Jiang, J. Multidisciplinary design optimization of adaptive wing leading edge. Sci. China Technol. Sci. 2013, 56, 1790-1797. [CrossRef]

23. Lyu, Z.; Martins, J.R.R.A. Aerodynamic Shape Optimization of an Adaptive Morphing Trailing Edge Wing. J. Aircr. 2015, 52, 1951-1970. [CrossRef]

24. Secanell, M.; Suleman, A.; Gamboa, P. Design of a Morphing Airfoil Using Aerodynamic Shape Optimization. AIAA J. 2006, 44, 1550-1562. [CrossRef]

25. Sugar Gabor, O.; Koreanschi, A.; Botez, R.M. Optimization of an Unmanned Aerial System' Wing Using a Flexible Skin Morphing Wing. SAE Int. J. Aerosp. 2013, 6, 115-121. [CrossRef]

26. De Gaspari, A.; Gilardelli, A.; Ricci, S.; Airoldi, A.; Moens, F. Design of a Leading Edge Morphing Based on Compliant Structures in the Framework of the CS2-AIRGREEN2 Project. In Proceedings of the ASME 2018 Conference on Smart Materials, Adaptive Structures and Intelligent Systems, San Antonio, TX, USA, 10-12 September 2018. [CrossRef]

27. Allmaras, S.R.; Johnson, F.T. Modifications and Clarifications for the Implementation of the Spalart-Allmaras Turbulence Model. In Proceedings of the Seventh International Conference on Computational Fluid Dynamics (ICCFD7), Big Island, HI, USA, 9-13 July 2012.

28. Menter, F. Two-equation eddy-viscosity turbulence models for engineering applications. AIAA J. 1994, 32, 1598-1605. [CrossRef]

29. Menter, F.R.; Smirnov, P.E.; Liu, T.; Avancha, R. A One-Equation Local Correlation-Based Transition Model. Flow Turbul. Combust. 2015, 95, 583-619. [CrossRef]

30. Menter, F.; Langtry, R.; Völker, S. Transition Modelling for General Purpose CFD Codes. Flow Turbul. Combust. 2006, 77, 277-303. [CrossRef]

31. Drela, M. XFOIL: An Analysis and Design System for Low Reynolds Number Airfoils. In Low Reynolds Number Airfoil Aerodynamics; Springer: Berlin/Heidelberg, Germany, 1989.

32. Comis Da Ronco, C.; Benini, E. A Simplex Crossover based evolutionary algorithm including the genetic diversity as objective. Appl. Soft Comput. 2013, 13, 2104-2123. [CrossRef]

33. Benini, E.; Venturelli, G.; Łaniewski, W. Comparison between pure and surrogate assisted evolutionary algorithms for multiobjective optimization. Front. Artif. Intell. Appl. 2016, 281, 229-242. [CrossRef]

34. Samareh, J.A. A Survey of Shape Parametrisation Techniques. In CEAS/AIAA/ICASE/NASA Langley International Forum on Aeroelasticity and Structural Dynamics 1999; National Aeronautics and Space Administration: Hampton, VA, USA, 1999.

35. Suzuki, H.; Rinoie, K.; Tezuka, A. Laminar Airfoil Modification Attaining Optimum Drag Reduction by Use of Airfoil Morphing. J. Aircr. 2010, 47, 1126-1132. [CrossRef]

36. Du, S.; Ang, H. Design and Feasibility Analyses of Morphing Airfoil Used to Control Flight Attitude. Strojniški Vestnik J. Mech. Eng. 2012, 58, 46-55. [CrossRef]

37. Kulfan, B.M. CST Universal Parametric Geometry Representation Method with Application to Supersonic Aircraft. In Proceedings of the Fourth International Conference on Flow Dynamics, Sendai, Japan, 26-28 September 2007.

38. Magrini, A.; Benini, E. Aerodynamic Optimization of a Morphing Leading Edge Airfoil with a Constant Arc Length Parameterization. J. Aerosp. Eng. 2018, 31, 04017093. [CrossRef]

39. Bettadapura, R.; Mashburn, T.; Crawford, R. Length-Constrained Bezier Curve Smoothing. Available online: https:/ / pdfs.semanticscholar.org/b96a/4066a532713d31fcd4e54cfd4e0c1511a0aa.pdf (accessed on 21 January 2019).

(c) 2019 by the authors. Licensee MDPI, Basel, Switzerland. This article is an open access article distributed under the terms and conditions of the Creative Commons Attribution (CC BY) license (http://creativecommons.org/licenses/by/4.0/). 